\title{
Yeast Rnt1p is required for cleavage of the pre-ribosomal RNA in the 3' ETS but not the 5' ETS
}

\author{
JOANNA KUFEL, BERNHARD DICHTL, ${ }^{1}$ and DAVID TOLLERVEY \\ Institute of Cell and Molecular Biology, University of Edinburgh, King's Buildings, Edinburgh EH9 3JR, United Kingdom
}

\begin{abstract}
We have reexamined the role of yeast RNase III (Rnt1p) in ribosome synthesis. Analysis of pre-rRNA processing in a strain carrying a complete deletion of the RNT1 gene demonstrated that the absence of Rnt1p does not block cleavage at site $A_{0}$ in the $5^{\prime}$ external transcribed spacers (ETS), although the early pre-rRNA cleavages at sites $A_{0}, A_{1}$, and $A_{2}$ are kinetically delayed. In contrast, cleavage in the $3^{\prime}$ ETS is completely inhibited in the absence of Rnt1p, leading to the synthesis of a reduced level of a $3^{\prime}$ extended form of the 25S rRNA. The $3^{\prime}$ extended forms of the pre-rRNAs are consistent with the major termination at site T2 $(+210)$. We conclude that Rnt1p is required for cleavage in the $3^{\prime}$ ETS but not for cleavage at site $A_{0}$. The sites of in vivo cleavage in the $3^{\prime}$ ETS were mapped by primer extension. Two sites of Rnt1p-dependent cleavage were identified that lie on opposite sides of a predicted stem loop structure, at +14 and +49. These are in good agreement with the consensus Rnt1p cleavage site. Processing of the $3^{\prime}$ end of the mature $25 \mathrm{~S}$ rRNA sequence in wild-type cells was found to occur concomitantly with processing of the $5^{\prime}$ end of the $5.8 \mathrm{~S}$ rRNA, supporting previous proposals that processing in ITS1 and the 3' ETS is coupled.
\end{abstract}

Keywords: endonuclease; RNA processing; RNase III; yeast

\section{INTRODUCTION}

Most functional RNA molecules are generated from precursor transcripts by posttranscriptional processing. Several exoribonucleases have been identified in prokaryotes and eukaryotes, whereas the number of known endonucleases is still very limited. In the yeast Saccharomyces cerevisiae only three endoribonucleases have been identified. Two of these are homologous ribonucleoprotein complexes, RNase $\mathrm{P}$ and RNase MRP, that cleave pre-tRNAs (reviewed by Altman et al., 1993) and site $A_{3}$ in the pre-rRNA, respectively (Lygerou et al., 1996). The other known endonuclease is Rnt1p.

Yeast Rnt1p was identified by sequence homology to bacterial RNase III (Abou Elela et al., 1996). Rnt1p is a double-strand specific ribonuclease that is required for the synthesis of several small nucleolar RNAs (snoRNAs) from large precursors (Chanfreau et al., 1998a, 1998b; Qu et al., 1999) and for 3' end matura-

Reprint requests to: David Tollervey, Institute of Cell and Molecular Biology, University of Edinburgh, King's Buildings, Edinburgh EH9 3JR, United Kingdom; e-mail: d.tollervey@ed.ac.uk.

${ }_{1}$ Present address: Biozentrum der Universität, Abteilung Zellbiologie, Klingelbergerstrasse 70, 4056 Basel, Switzerland. tion of the $\mathrm{U} 1, \mathrm{U} 2$, and $\mathrm{U} 5$ small nuclear RNAs (snRNAs) (Chanfreau et al., 1997; Abou Elela \& Ares, 1998; Seipelt et al., 1999). The initial functions reported for yeast Rnt1p were, however, in the processing of the prerRNA (Abou Elela et al., 1996).

The eukaryotic $18 \mathrm{~S}, 5.8 \mathrm{~S}$, and $25 \mathrm{~S} / 28 \mathrm{~S}$ rRNAs are transcribed as a single precursor molecule that undergoes complex posttranscriptional processing to remove the external transcribed spacers (5' ETS and 3' ETS) and internal transcribed spacers (ITS1 and ITS2). This process involves several exonucleolytic and endonucleolytic steps (Venema \& Tollervey, 1995; Fig. 1B) and is largely carried out in the nucleolus. The two earliest processing events, in the $3^{\prime}$ ETS and at site $A_{0}$ in the $5^{\prime}$ ETS, were reported to be inhibited in a temperature-sensitive rnt1-1 strain in vivo (Abou Elela et al., 1996). In addition, model $3^{\prime}$ ETS and 5' ETS substrates comprising stem-loop structures were specifically cleaved in vitro by the recombinant GST-Rnt1p fusion protein. This strongly suggested that Rnt1p directly cleaved these two sites. Since RNase III in Escherichia coli also participates in pre-rRNA processing (King et al., 1984), this result greatly influenced models for the evolutionary origins of the eukaryotic pre-rRNA processing machinery. 
A

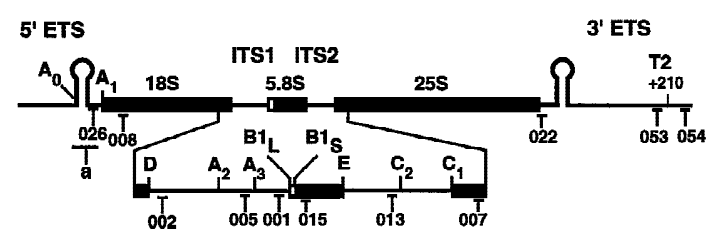

B

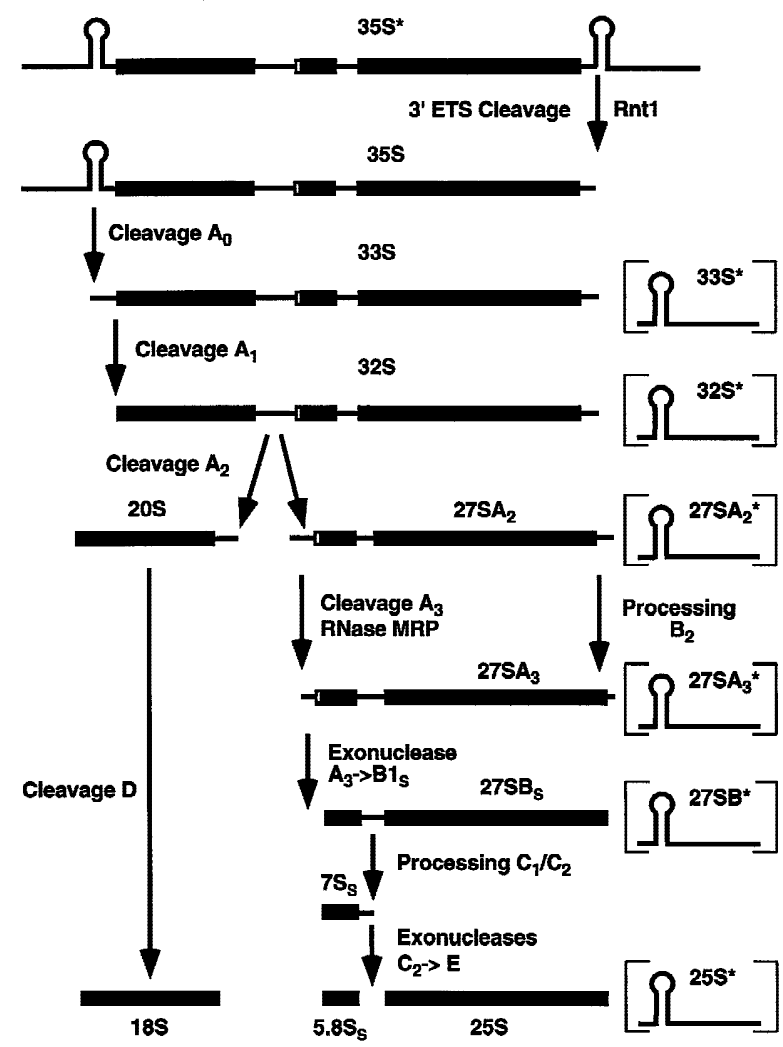

FIGURE 1. Structure of the pre-rRNA and processing pathway in Saccharomyces cerevisiae. A: Structure of the 35S pre-rRNA with the location of oligonucleotide probes used for hybridization and primer extension. Probe a is a riboprobe complementary to the $A_{0}-A_{1}$ fragment. Positions of cleavage sites are shown in upper case. The putative transcription termination site $\mathrm{T} 2$ at position +210 is indicated. B: Major pre-rRNA processing pathway. 35S pre-rRNA undergoes sequential processing reaction to generate the mature rRNAs. Cleavage in the $3^{\prime}$ ETS and snoRNP-dependent cleavage at site $A_{0}$ in the $5^{\prime}$ ETS generate 33S pre-rRNA, which is further processed at other snoRNP-dependent cleavage sites, $A_{1}$ in the $5^{\prime}$ ETS at the $5^{\prime}$ end of the $18 \mathrm{~S}$ rRNA, giving rise to $32 \mathrm{~S}$ pre-rRNA, and $\mathrm{A}_{2}$ in ITS1, yielding the $20 S$ and $27 S A_{2}$ pre-RNAs. $A_{2}$ cleavage separates the pre-RNAs destined to form the small and large ribosomal subunit rRNAs. The $20 \mathrm{~S}$ precursor is then endonucleolytically cleaved at site $D$ to generate the mature $18 \mathrm{~S}$ rRNA. The $27 \mathrm{SA}_{2}$ pre-rRNA is processed by two alternative pathways, giving rise to two forms of $5.8 \mathrm{~S} \mathrm{rRNA}$, the major $5.8 \mathrm{~S}_{\mathrm{S}}$ form and minor $5.8 \mathrm{~S}_{\mathrm{L}}$ form. Only the major pathway leading to the synthesis of $5.8 \mathrm{~S}_{\mathrm{S}}$ is shown. In this pathway, $27 S A_{2}$ is cleaved by RNase MRP at site $A_{3}$ to yield the $27 \mathrm{SA}_{3}$, which is trimmed by $5^{\prime} \rightarrow 3^{\prime}$ exonucleases Rat 1 and Xrn1 to site $B 1_{s}$, generating the $5^{\prime}$ end of the $27 \mathrm{SB}_{S}$ pre-rRNA and mature $5.8 S_{S} r R N A$. An alternative pathway leads to cleavage at site $B 1_{L}$, the $5^{\prime}$ end of the $27 S_{L}$ pre-rRNA and mature $5.8 S_{L}$ rRNA. The processing of both 27SB species is identical. Processing at sites $C_{1}$ and $C_{2}$ separates the mature 25S rRNA and the 7S pre-RNA, which is converted to $5.8 \mathrm{~S}$ rRNA by the exosome complex of $3^{\prime} \rightarrow 5^{\prime}$ exonucleases. $3^{\prime}$ extended species denoted with * in the text are depicted in brackets alongside their respective counterparts.
Cleavage at site $A_{0}$ also requires base pairing between the U3 snoRNA and the 5' ETS (Beltrame \& Tollervey, 1995). We therefore investigated the relationship between U3 and Rnt1p. In the course of this work we realized that a strain completely lacking Rnt1p is, in fact, able to efficiently cleave site $A_{0}$. In contrast, cleavage in the $3^{\prime}$ ETS is inhibited, as previously reported (Abou Elela et al., 1996), although the sites of in vivo Rnt1p cleavage do not match the previously reported site of in vitro cleavage.

\section{RESULTS}

\section{Cleavage at $A_{0}$ in the $5^{\prime}$ ETS does not require Rnt1p}

Because cleavage at site $A_{0}$ was reported to require the function of both the U3 snoRNP (Hughes \& Ares, 1991) and Rnt1p (Abou Elela et al., 1996), we tested for physical and/or functional links between these two components. The RNT1 gene was originally reported to be essential for viability (Abou Elela et al., 1996). We therefore constructed an $\mathrm{N}$-terminal $\mathrm{His}_{8}$-epitope-tagged allele of RNT1 under the control of the repressible GAL10 promoter and integrated this at the RNT1 locus in a haploid yeast strain (GAL::rnt1; see Materials and Methods). Correct integration of the HIS3-GAL10 fragment was confirmed by PCR and Southern hybridization (data not shown). No coprecipitation of the U3 snoRNA with the His ${ }_{8}$-Rnt1p fusion was detected (data not shown). Unexpectedly, we noted that cleavage at site $A_{0}$ in the 5' ETS continued on depletion of Rnt1p by growth of the GAL::rnt1 strain on glucose medium (Fig. 2, lanes 4-6).

During the course of this work it was reported that RNT1 was not essential for viability, although its disruption resulted in a strong growth defect (Abou Elela \& Ares, 1998; Chanfreau et al., 1998b). Two wild-type strains and two rnt1- $\Delta$ sister strains carrying complete deletions of RNT1 (kindly provided by Guillaume Chanfreau) were used for further studies. The requirement for Rnt $1 p$ in cleavage at site $A_{0}$ was analyzed by primer extension on RNA extracted from the GAL::rnt1 strain and the isogenic wild-type strain (WT) shifted from permissive, RSG medium to repressive, glucose medium (Fig. 2, lanes 1-6) and from $r n t 1-\Delta$ and RNT1 sister strains (Fig. 2, lanes 8-11). Primer extension through $A_{0}$ was performed with two different primers; primer 008 is complementary to the $5^{\prime}$ region of mature 18S rRNA whereas primer 026 hybridizes to the $5^{\prime}$ ETS/18S boundary (see Fig. 1A). The two primers show subtly different aspects of the pre-rRNA population. Primer 026 (Fig. 2, row III) shows the level of pre-rRNA species that are cleaved at $A_{0}$, primarily the $33 S$ prerRNA (or $33 S^{*}$ pre-rRNA in the case of the rnt1- $\Delta$ mutants; see below), compared to total RNA. Primer 008 (Fig. 2, row II) largely reveals the ratio between the 


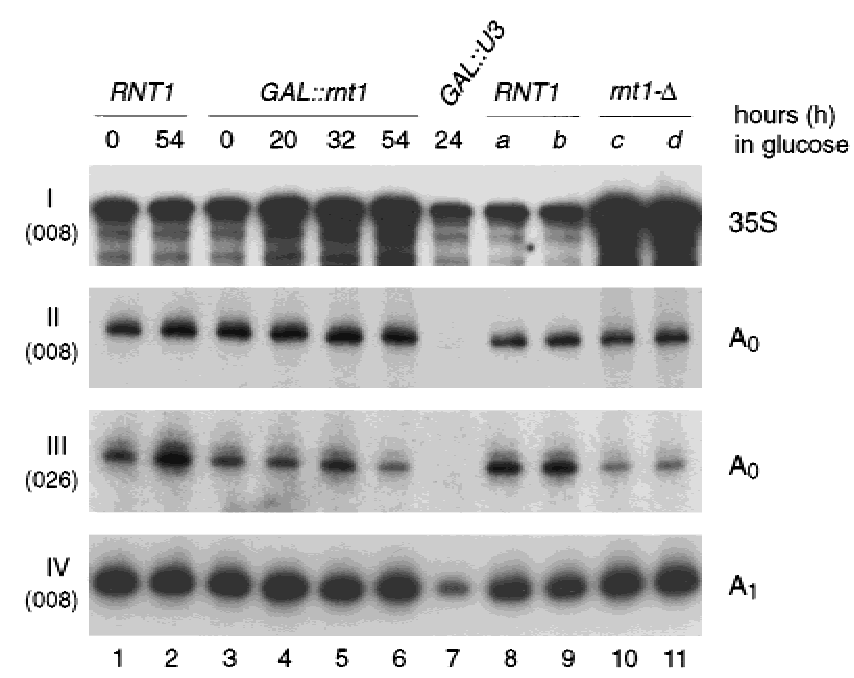

FIGURE 2. Strains lacking Rnt1p continue to cleave site $A_{0}$. Primer extension analysis through $5^{\prime}$ ETS of the pre-rRNA. I, II and IV: primer complementary to the mature 18S rRNA (oligonucleotide 008). III: primer complementary to the 5' ETS/18S boundary (oligonucleotide 026). Numbers of oligonucleotide probes are given in parentheses. The GAL::rnt1 strain and the isogenic wild-type strain (WT) were grown in RSG medium ( $0 \mathrm{~h}$ ) and transferred to glucose medium for the times indicated $(9-54 \mathrm{~h})$. The GAL::U3 strain (JH84) was grown in galactose medium and transferred to glucose medium for $24 \mathrm{~h}$. The RNT1 (spores a and b) and $r n t 1-\Delta$ (spores $\mathrm{c}$ and d) sister strains were grown in glucose medium at $30^{\circ} \mathrm{C}$.

$33 S / 33 S^{*}$ pre-rRNA and the $18 S$ rRNA. No clear alteration in the primer extension stop at site $A_{0}$ was observed in the rnt1- $\Delta$ strain using primer 008, showing that cleavage at site $A_{0}$ continued in the absence of Rnt1p. A reduced, but still substantial, stop at $A_{0}$ was observed using primer 026 . This may indicate that in the mutant strain some cleavage at $A_{1}$ occurs prior to cleavage at $A_{0}$. These results were in marked contrast to a $G A L:: U 3$ strain in which SNR17B (encoding U3B) was disrupted and SNR17A encoding U3A snoRNA has been placed under the control of the GAL10 promoter (kindly provided by John Hughes). As previously reported (Hughes \& Ares, 1991), transfer of this strain from galactose to glucose medium for $24 \mathrm{~h}$ resulted in a drastic reduction in the primer extension stop at $A_{0}$ (Fig. 2, rows II and III, lane 7).

The primer extension stop corresponding to the $5^{\prime}$ end of the $35 \mathrm{~S}$ and $35 \mathrm{~S}^{*}$ increased on depletion of Rnt1p and in the rnt1- $\Delta$ strains (Fig. 2, row I, primer 008), showing a delay in processing of the $35 S^{*}$ prerRNA. The identical result was observed with primer 026 (data not shown). Also shown is the primer extension stop at $A_{1}$, the $5^{\prime}$ end of the $18 \mathrm{~S}$ rRNA, which is almost unchanged in the absence of Rnt1p but is depleted in the GAL::U3 strain (Fig. 2, row IV, lane 7).

The previously reported block in cleavage at site $A_{0}$ in the rnt1-1 strain was temperature-sensitive (Abou Elela et al., 1996). In the rnt1- $\Delta$ strains, the growth defect is also severely aggravated at $37^{\circ} \mathrm{C}$ (Chanfreau et al., 1998b). To determine whether $A_{0}$ cleavage is temperature sensitive in the rnt1- $\Delta$ strain, RNA was extracted during growth at $25^{\circ} \mathrm{C}$ (Fig. $3 \mathrm{~A}$, lane 3 ) and following transfer to $37^{\circ} \mathrm{C}$ (Fig. $3 \mathrm{~A}$, lanes 4-6). The primer extension stop at $A_{0}$ observed with oligo 008 was unaffected by growth of the $r n t 1-\Delta$ strain at $37^{\circ} \mathrm{C}$, whereas a reduced primer extension stop at $A_{0}$ in the $r n t 1-\Delta$ strain was detected using primer 026 (Fig. 3A, row III, lanes 4-6), even at late time points. A similar reduction was observed in the primer extension stop at site $A_{2}$, the $5^{\prime}$ end of the $27 S A_{2}$ and $27 S A_{2}^{*}$ pre-rRNAs, consistent with a general delay in the early pre-rRNA processing steps at $A_{0}, A_{1}$, and $A_{2}$.

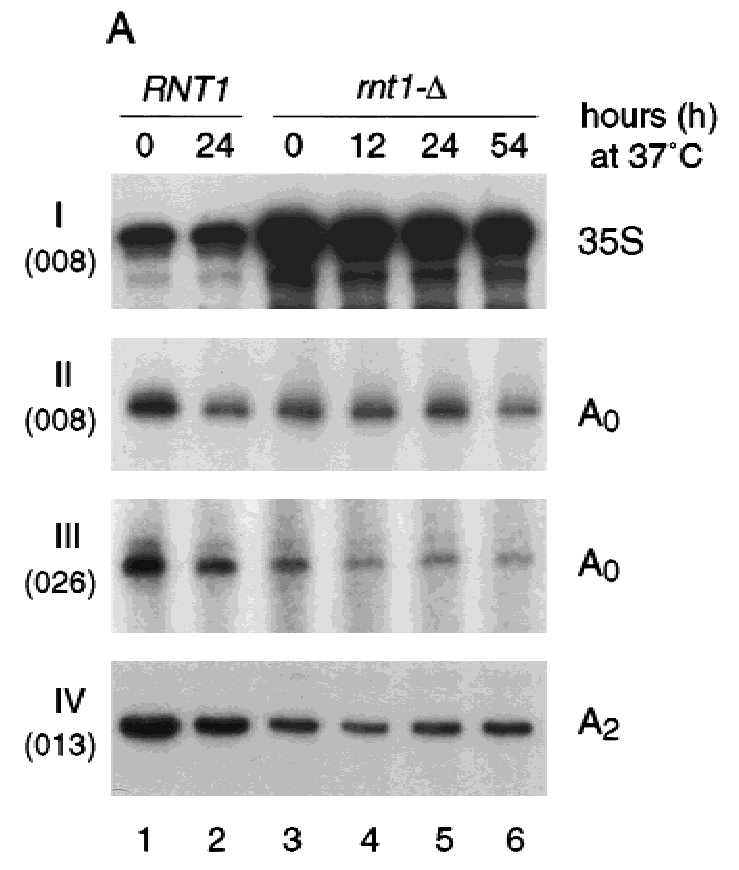

B

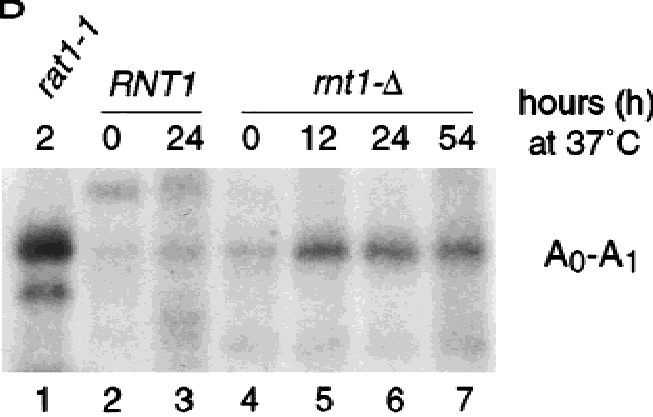

FIGURE 3. Cleavage at site $A_{0}$ is not temperature sensitive in an rnt1- $\Delta$ strain. A: Primer extension analysis through the 5' ETS (I-III) and ITS1 (IV) of the pre-rRNA. I and II: primer complementary to the mature 18S rRNA (oligonucleotide 008). III: primer complementary to the $5^{\prime}$ ETS/18S boundary (oligonucleotide 026). IV: primer complementary to the $5^{\prime}$ region of ITS2 (oligonucleotide 013 ). Numbers of oligonucleotide probes are given in parentheses. B: Northern analysis of the accumulation of the excised $A_{0}-A_{1}$ fragment with probe $a$. RNT1 and $r n t 1-\Delta$ sister strains were pregrown at $25^{\circ} \mathrm{C}(0 \mathrm{~h})$ and transferred to $37^{\circ} \mathrm{C}$ for the times indicated $(12-54 \mathrm{~h})$. The rat1-1 strain was grown at $25^{\circ} \mathrm{C}$ and transferred to $37^{\circ} \mathrm{C}$ for $2 \mathrm{~h}$. 
To confirm the continued cleavage at site $A_{0}$, the excised $A_{0}-A_{1}$ fragment was examined by Northern hybridization using a riboprobe complementary to the region between sites $A_{0}$ and $A_{1}$. The excised fragment was readily detected in the $r n t 1-\Delta$ strain grown either at $25^{\circ} \mathrm{C}$ (Fig. 3B, lane 4) or shifted to $37^{\circ} \mathrm{C}$ (Fig. 3B, lanes 5-7). Indeed, the level of the $A_{0}-A_{1}$ fragment was increased in the rnt1- $\Delta$ strain at $37^{\circ} \mathrm{C}$. If cleavage at site $A_{1}$ is occurring prior to $A_{0}$ in the rnt1- $\Delta$ strain, as suggested by the primer extension data, this may affect the kinetics of degradation of the excised fragment. To confirm the identity of this band, RNA was extracted from a rat1-1 strain, which accumulates the $A_{0}-A_{1}$ fragment (Petfalski et al., 1998), $2 \mathrm{~h}$ after transfer to $37^{\circ} \mathrm{C}$ (Fig. 3B, lane 1).

The absence of the RNT1 mRNA from the mutant strains was confirmed by Northern hybridization (Fig. 4, row $\mathrm{XI}$ ). No RNT1 mRNA was detected in the rnt1- $\Delta$ strains (Fig. 4, row XI, lanes 10 and 11). In the GAL:: rnt1 strain, some overexpression of RNT1 was observed in permissive RSG medium (Fig. 4, row XI, lane 3 ), and the mRNA was strongly depleted following transfer to glucose medium (Fig. 4, row XI, lanes 4-7). The faint band visible above the RNT1 mRNA in lanes $6-7$ is probably due to cross-hybridization to mature 18S rRNA. The difference in the position of RNT1 mRNA between the two wild-type strains (designated $W T$ and RNT1) presumably reflects strain heterogeneity. Rnt1p is also required for processing of the dimeric precursor to the snR190 and U14 snoRNAs (Chanfreau et al., 1998b). The inhibition of this processing was observed in the rnt1- $\Delta$ strains and in the GAL::rnt1 strain following growth on glucose medium (data not shown).

We conclude that cleavage at site $A_{0}$ does not require Rnt1p.

\section{Rnt1p is required for processing in the $3^{\prime}$ ETS}

The effects of the depletion or absence of Rnt $1 p$ on other pre-rRNA processing reactions were assessed by Northern hybridization (Fig. 4). In general, the same defects were observed in the GAL::rnt1 strain following transfer to glucose medium and in the rnt1- $\Delta$ strain. The phenotypes were, however, more marked in the rnt1- $\Delta$ strains that completely lack Rnt1p. The lower levels of pre-rRNAs seen in the strains grown in RSG medium (Fig. 4, lanes 1 and 3) are due to the effects of nutritional shift down on pre-rRNA transcription.

Rnt1p was reported to cleave the pre-rRNA in the $3^{\prime}$ ETS (Abou Elela et al., 1996). Consistent with this, we detect $3^{\prime}$ extended forms of pre-rRNA species that normally terminate at the $3^{\prime}$ ETS (35S, 27SA, and 27SB) as well as the 25S rRNA. The extended species are indicated as $35 S^{*}, 32 S^{*}, 27 \mathrm{SA}_{2}^{*}, 27 \mathrm{SB}^{*}$, and $25 \mathrm{~S}^{*}$ (see also Fig. 1B). Little, if any, of the wild-type 35S, 27S, or 25S RNAs are detected, indicating that processing of
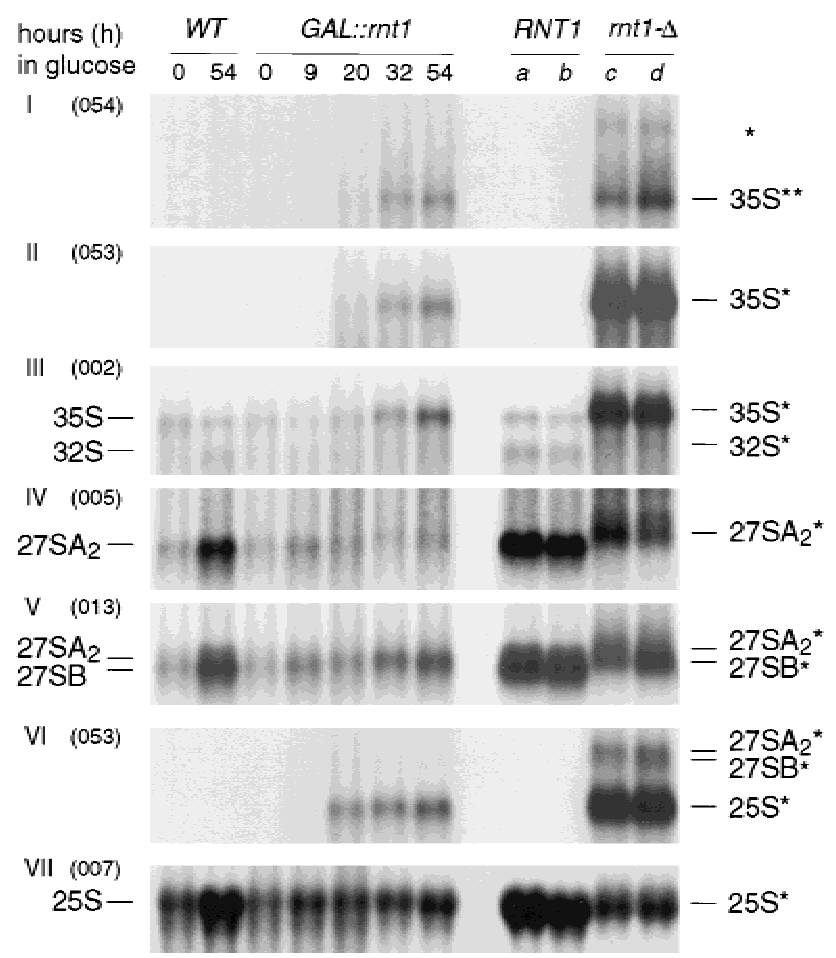

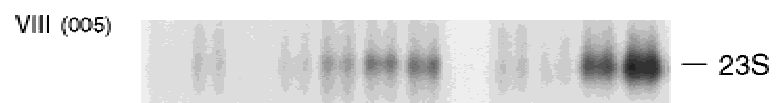
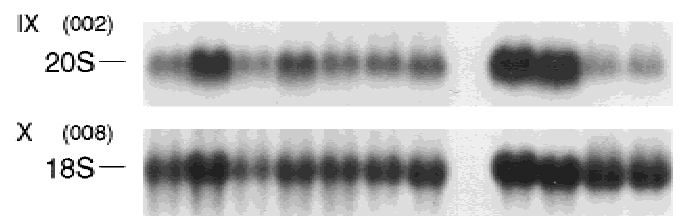

$\mathrm{XI}$

RNT1 mRNA

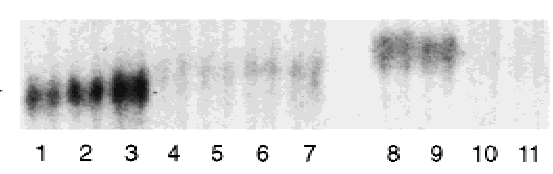

FIGURE 4. Rnt 1 is required for processing of the $3^{\prime}$ ETS. Strains were grown as described for Figure 2. RNA was separated on a $1.2 \%$ agarose gel and analyzed by Northern hybridization. Only regions of each Northern are presented; together these show all high molecular weight pre-rRNAs detected. I: hybridization with oligo 054 complementary to the 3' ETS downstream of position 264; II: hybridization with oligo 053 complementary to the $3^{\prime}$ ETS downstream of position 180; III: hybridization with oligo 002 complementary to the $5^{\prime}$ region of ITS1; IV and VIII: hybridization with oligo 005 complementary to the central region of ITS1, between sites $\mathrm{A}_{2}$ and $\mathrm{A}_{3}$; V: hybridization with oligo 013 complementary to the $5^{\prime}$ region of ITS2; VI: hybridization with oligo 053 complementary to the $3^{\prime}$ ETS downstream of position 180; VII: hybridization with oligo 007 complementary to the mature 25S rRNA; IX: hybridization with oligo 002 complementary to the $5^{\prime}$ region of ITS1; $X$ : hybridization with oligo 008 complementary to the mature $18 \mathrm{~S}$ rRNA; XI: hybridization with a probe complementary to the RNT1 mRNA. The positions of the wild-type RNAs are indicated on the left. Species marked with * represent $3^{\prime}$ extended intermediates and are indicated on the right. Numbers of oligonucleotide probes are given in parentheses.

the $3^{\prime}$ ETS does not occur in the absence of Rnt1p. The $3^{\prime}$ extended species were detected by probe 053 , hybridizing to the $3^{\prime}$ ETS at +180 (Fig. 4, row II), but not by probe 054 hybridizing at +264 (Fig. 4, row I). 
Probe 022, hybridizing across the $25 \mathrm{~S}$ rRNA/3' ETS junction, did not give a stronger signal than probe 053 (data not shown), indicating that the major transcripts in the rnt1- $\Delta$ mutants terminate between +180 and +264 . This suggests that they terminate at site T2 (position +210 ) (Veldman et al., 1980; Kempers-Veenstra et al., 1986; van der Sande et al., 1989) rather than the termination site identified in vitro at +108 , close to the Reb1p binding site (Lang \& Reeder, 1993). Much lower levels of the larger $35 S^{* *}$ RNA were detected with oligo 054 in the rnt1- $\Delta$ strain (note that the exposure of the Northern shown in Fig. 4, row I, was 2.5-fold longer than that shown in Fig. 4, row II). $35 S^{* *}$ extends beyond +210 , most likely because of read-through of T2, possibly to termination site T3A at +690 (van der Sande et al., 1989). For reasons that are not clear, the ratio of $35 S^{* *}$ to $35 S^{*}$ is higher in the GAL::rnt1 strain than in the rnt1- $\Delta$ strain. The larger species marked * (Fig. 4, row I) has not been further characterized, but it may extend through the nontranscribed spacer region to termination site $\mathrm{Tp}$, reported to lie $300 \mathrm{nt}$ upstream of the next rDNA transcription unit (van der Sande et al., 1989).

We conclude that pre-rRNA transcription normally terminates in the $3^{\prime}$ ETS between +180 and +264 (most probably at +210 ) with lower levels of read-through transcripts. These species are presumably not detected in wild-type cells because Rnt1p cleaves the nascent pre-rRNAs cotranscriptionally.

The 25S* rRNA was underaccumulated in both the GAL::rnt1 strain grown on glucose medium and in the rnt1- $\Delta$ strains (Fig. 4, row VII), compared to the $25 \mathrm{~S}$ rRNA in wild-type strains. The level of the $27 \mathrm{SB}^{*}$ prerRNA (Fig. 4, row V) was also reduced compared to the wild-type species, indicating that synthesis of the 25S rRNA is inhibited in the strains lacking Rnt1p. The 20S and $27 \mathrm{SA}_{2}$ pre-rRNAs are both generated by cleavage at site $A_{2}$ (see Fig. 1B). In the rnt1- $\Delta$ strains, the $27 S A_{2}^{*}$ (Fig. 4, row IV) is not, however, reduced as much as $20 S^{*}$ (Fig. 4, row IX), indicating that processing of $27 S A_{2}^{*}$ to $27 \mathrm{SB}^{*}$ is delayed. In the absence of Rnt1p, the $3^{\prime}$ extended pre-rRNAs therefore appear to be inefficiently processed.

The timing of the processing of the $3^{\prime}$ end of the mature 25S rRNA in wild-type cells was assessed using probe 022 complementary to the $25 \mathrm{~S}$ rRNA/3' ETS junction (Fig. 5, lane 1). This probe detects RNA species carrying extensions beyond the mature end of $25 \mathrm{~S}$ rRNA. 35S, 32S, and $27 \mathrm{SA}_{2}$ pre-rRNAs were detected in wild-type cells using this probe, whereas no $27 \mathrm{SB}$ pre-rRNA was observed (compare hybridization with probe 022 to hybridizations with probes 005 and 013 detecting $27 \mathrm{SA}_{2}$ and $27 \mathrm{SB}$ ). This indicates that processing of the $3^{\prime}$ end of $25 S$ rRNA occurs concomitantly with processing at site $B_{1}$ at the $5^{\prime}$ end of the mature 5.8S rRNA. The faint band visible below the $27 \mathrm{SA}_{2} \mathrm{RNA}$ in Figure 5, lane 1 , is probably due to cross-hybridization with mature $25 \mathrm{~S}$ rRNA, although

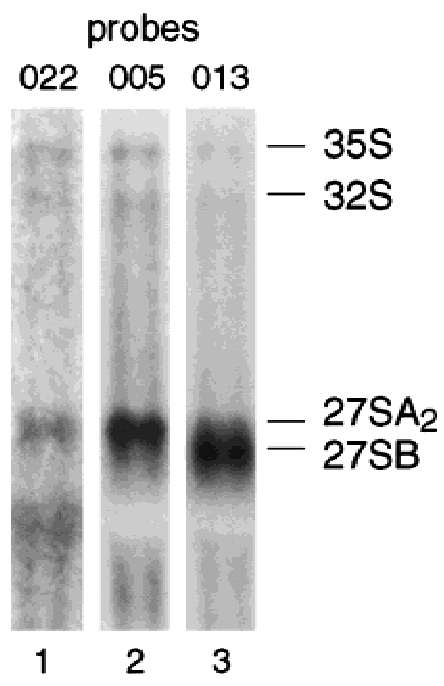

FIGURE 5. Timing of the processing of the $3^{\prime}$ end of the mature $25 \mathrm{~S}$ rRNA. RNA was extracted from the wild-type strain, separated on a $1.2 \%$ agarose gel, and analyzed by Northern hybridization. Numbers of oligonucleotide probes are indicated on the top. Lane 1: hybridization with oligo 022 complementary to the $25 \mathrm{~S}$ rRNA/3' ETS boundary. Lane 2: hybridization with oligo 005 complementary to the central region of ITS1, between sites $A_{2}$ and $A_{3}$. Lane 3: hybridization with oligo 013 complementary to the $5^{\prime}$ region of ITS2.

we cannot exclude the possibility that a small fraction of the $5^{\prime}$ matured 25S rRNA population retains the $3^{\prime}$ extension. These results are in agreement with the previous report that a mixed pre-rRNA fraction containing $27 \mathrm{SA}_{2}$ and $27 \mathrm{SB}$ had a heterogeneous $3^{\prime}$ end (quoted in Veldman et al., 1981), also indicating that $3^{\prime}$ processing occurs between 27SA and 27SB.

Relative use of the alternative ITS1 processing pathways was assessed by primer extension and Northern hybridization (Fig. 6). There was little alteration in the level of the $27 \mathrm{SA}_{3}$ pre-rRNA as shown by the primer extension stop at $A_{3}$ (Fig. $6 B$, row I). Consistent with this, the ratio between the mature $5.8 S_{S}$ and $5.8 S_{L}$ rRNAs was not clearly different in the rnt1- $\Delta$ and wildtype strains, as judged by quantitation of a Northern hybridization (Fig. 6A). However, the ratio of $27 \mathrm{SB}_{\mathrm{S}}$ to $27 \mathrm{SB}_{\mathrm{L}}$ was decreased in the rnt1- $\Delta$ strain, particularly at later time points at $37^{\circ} \mathrm{C}$ (Fig. $6 \mathrm{~B}$, row II). Both forms of the 27SB pre-rRNA were reduced, consistent with the Northern hybridization. In view of the lack of effects on $\mathrm{A}_{3}$ cleavage on the $5.8 \mathrm{~S}_{\mathrm{S}}: 5.8 \mathrm{~S}_{\mathrm{L}}$ ratio, the increase in the relative level of $27 \mathrm{SB}_{\mathrm{L}}$ may reflect a mild delay in its processing.

In addition to the defects in 25S rRNA synthesis, the early pre-rRNA processing steps were delayed in the $G A L:: r n t 1$ and $r n t 1-\Delta$ strains. Consistent with the primer extension data, the $35 S^{*}$ precursor was strongly accumulated compared to the wild-type 35S (Fig. 4, row III) and the aberrant 23S RNA was detected, accompanied by a reduced level of the 20S pre-rRNA. The 23S RNA extends from the $5^{\prime}$ end of the $5^{\prime}$ ETS to site $A_{3}$ and is 
A
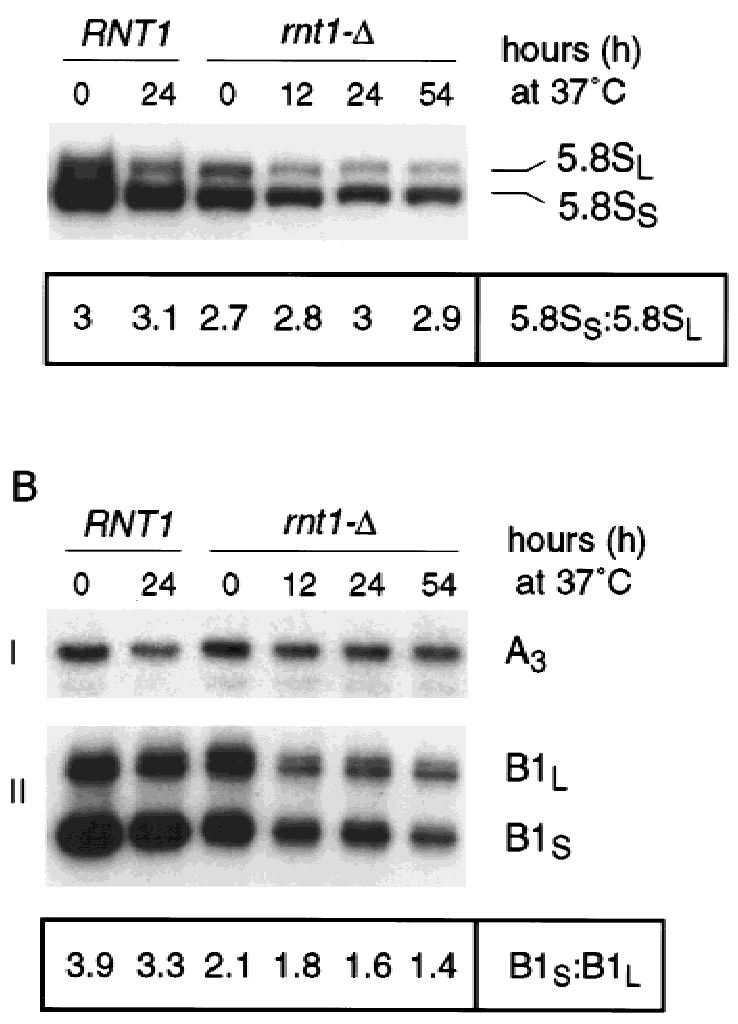

FIGURE 6. Effects of loss of Rnt1p on the 5.8S rRNA processing pathways. Strains were grown as described for Figure 3. RNA was extracted from $R N T 1$ and $r n t 1 \Delta$ sister strains grown at $25^{\circ} \mathrm{C}$ or following transfer to $37^{\circ} \mathrm{C}$ for the times indicated. The ratios of the steady-state levels of $5.8 \mathrm{~S}_{\mathrm{S}}: 5.8 \mathrm{~S}_{\mathrm{L}}$ and of the primer extension stops $B 1_{S}: B 1_{L}$ were quantified with a Phosphorlmager (Molecular Dynamics) and the values obtained are indicated. A: RNA was separated on a $6 \%$ polyacrylamide gel and analyzed by Northern hybridization with oligo 015 against the mature 5.8S rRNA species. Two forms of the mature $5.8 \mathrm{~S}$ rRNAs, $5.8 \mathrm{~S}_{\mathrm{S}}$ and $5.8 \mathrm{~S}_{\mathrm{L}}$, are indicated. B: RNA was analyzed by primer extension using oligo 013 . I: primer extension stop at $A_{3}$. II: primer extension stops at $B 1_{L}$ and $B 1_{S}$. Note that the exposure shown for I is approximately fivefold longer than for II.

the product of cleavage at site $A_{3}$ in the absence of prior cleavage at sites $A_{0}, A_{1}$, or $A_{2}$. However, the level of the 18S rRNA was unaltered in the rnt1- $\Delta$ or GAL:: rnt1 strains (Fig. 4, row X), in contrast to the effects on accumulation of the $25 \mathrm{~S}$ (Fig. 4, row VII) and 5.8S rRNAs (Fig. 6).

To map the sites of Rnt1p cleavage in the $3^{\prime}$ ETS in vivo, we performed primer extension using primer 053, hybridizing to the $3^{\prime}$ ETS at +180 . Total RNA was extracted from $r n t 1-\Delta$ and RNT1 sister strains grown at $30^{\circ} \mathrm{C}$. Primer extension stops were detected in the wildtype strain between positions $+14 /+15$ and $+49 /+50$ (Fig. 7A, lane 3). These stops are absent in the primer extension reaction for the $r n t 1-\Delta$ strain (Fig. 7A, lane 2) and we conclude that they correspond to the cleavage sites by Rnt1p. A much stronger primer extension signal was obtained for rnt1- $\Delta$ strain (the exposure for Fig. $7 \mathrm{~A}$, lane 3 , is $\sim 6$ times longer than for Fig. $7 \mathrm{~A}$, lane 2) reflecting the high abundance of $3^{\prime}$ extended pre-rRNAs in this strain. The $5^{\prime} \rightarrow 3^{\prime}$ exonuclease Rat1p participates in the degradation of several excised prerRNA spacer fragments that accumulate in the rat1-1 strain (Petfalski et al., 1998). RNA was extracted from the rat1-1 strain following transfer to $37^{\circ} \mathrm{C}$ for $2 \mathrm{~h}$ (Fig. 7A, lane 1). In this strain the primer extension stops between positions $+14 /+15$ and $+49 /+50$ are much stronger than in the wild-type (the exposure for Fig. $7 \mathrm{~A}$ lane 3 , is $\sim 6$ times longer than for Fig. 7A, lane 1), strongly indicating that these stops represent the $5^{\prime}$ ends of the cleaved pre-rRNA spacer fragments.

Unexpectedly, the sites mapped in vivo did not correspond to the site of cleavage previously mapped in vitro between residues $+21 /+22$ using recombinant Rnt1p and a model substrate (Abou Elela et al., 1996). However, the use of a purer enzyme preparation has now allowed in vitro cleavage at sites that are identical to those identified in vivo (M. Ares, pers. comm.).

\section{DISCUSSION}

We report the analysis of pre-rRNA processing in strains lacking Rnt1p. Cleavage in the 3' ETS was found to be blocked in the rnt1- $\Delta$ strain, as previously reported for the rnt1-1 strain (Abou Elela et al., 1996), leading to the synthesis of $3^{\prime}$ extended forms of the pre-rRNAs and $25 S$ rRNA. In contrast, the three early pre-rRNA cleavages at sites $A_{0}, A_{1}$, and $A_{2}$ were not blocked in the absence of Rnt1p, although they were delayed, leading to accumulation of the $35 S^{*}$ pre-rRNA. It is notable that many mutants defective in the synthesis of the 60S ribosomal subunit show defects in the early pre-rRNA processing steps that are similar to those observed in the rnt1- $\Delta$ strains (see, for example, Bergès et al., 1994; Weaver et al., 1997; Zanchin et al., 1997; Daugeron \& Linder, 1998; de la Cruz et al., 1998a, 1998b; Kressler et al., 1998). All of these mutations are characterized by underaccumulation of the $5.8 \mathrm{~S}$ and $25 \mathrm{~S}$ rRNAs and $60 S$ ribosomal subunits. Cleavage at sites $A_{0}, A_{1}$, and $A_{2}$ is inhibited, with accumulation of the $35 S$ pre-rRNA, appearance of the 23S rRNA, and reduced levels of the $20 \mathrm{~S}$ pre-rRNA. This is not, however, accompanied by reduced synthesis of mature 18S rRNA, showing that the early cleavages are delayed rather than being blocked. In cells lacking Rnt1p, defective processing in the $3^{\prime}$ ETS may result in misassembly of the 60S ribosomal subunits.

In addition to its role in processing the pre-rRNA, Rnt1p is required for the processing of the dicistronic snR190-U14 precursor, as well as several other snoRNAs (Chanfreau et al., 1998a, 1998b; Qu et al., 1999). In the absence of Rnt1p, U14 is present as the unprocessed snR190-U14 transcript (Chanfreau et al., 1998b; this work) and may well not be fully active. Genetic depletion of U14 blocked pre-rRNA cleavage at sites $A_{1}$ and $A_{2}$, while cleavage at site $A_{0}$ was delayed 


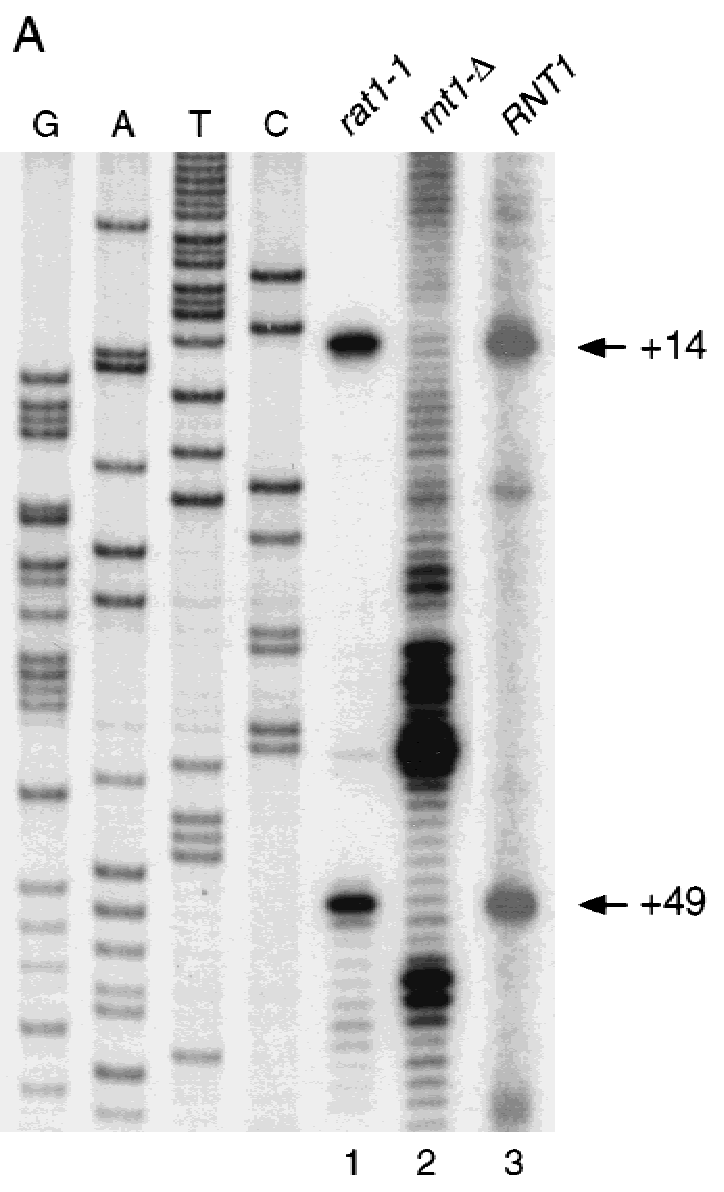

(Li et al., 1990), and the inhibition of synthesis of mature U14 in the rnt1- $\Delta$ strain may contribute to the delay in the early pre-rRNA processing reactions.

Rnt1p was also reported to be involved in the formation of $3^{\prime}$ end of the spliceosomal snRNAs, U1, U2, and U5 (Chanfreau et al., 1997; Abou Elela \& Ares, 1998; Seipelt et al., 1999). However, no defect in the splicing of the actin pre-mRNA was observed in the $r n t 1-\Delta$ strains (data not shown).

The effects on pre-rRNA processing of deletions in cis in the 3' ETS that destroyed the hairpin structure that is recognized by Rnt1p have also been investigated. Like the absence of Rnt1p in trans, these deletions inhibited processing in the $3^{\prime}$ ETS leading to $3^{\prime}$ extended forms of the pre-rRNAs and the 25S rRNA (Allmang \& Tollervey, 1998). However, the mutations in cis also inhibited pre-rRNA cleavage in ITS1 at site $A_{3}$, resulting in an increased $5.8 \mathrm{~S}_{\mathrm{L}}: 5.8 \mathrm{~S}_{\mathrm{S}}$ ratio. It was speculated that the inhibition of cleavage at $A_{3}$ was due to the coupling of the pre-rRNA cleavages in the $3^{\prime}$ ETS and ITS1 (Allmang \& Tollervey, 1998). The differences between the deletion of RNT1 and deletion of the cleavage site indicate that such coupling is not provided by Rnt1p. In contrast, deletion of the $3^{\prime}$ ETS did not detectably delay processing of the $5^{\prime}$ ETS. This difference may be due to the nature of the $3^{\prime}$ extensions. In the rnt1- $\Delta$ mutants, the extension is the native $3^{\prime}$ ETS, whereas the pre-rRNA deletion constructs have the RNA polymerase II GAL-terminator region. Conceivably the presence of the 3' ETS might be recognized by a quality control system as a sign that correct $3^{\prime}$ processing had not occurred, leading to a delay in the early processing reactions, whereas the GAL-terminator is unlikely to elicit such a response. Support for the coupling of processing in ITS1 and the $3^{\prime}$ ETS is provided by the observation that processing of the $3^{\prime}$ end of the $25 \mathrm{~S}$ and $5^{\prime}$ end of the $5.8 \mathrm{~S}$ occurs concomitantly.

Rnt1p, like bacterial RNase III, cleaves both strands of an imperfect stem-loop structure (Court, 1993; Abou Elela et al., 1996; Chanfreau et al., 1998a). Several substrates for Rnt1p have been identified. A common feature of these is a tetraloop with a consensus sequence AGNN closing the stem, which is located 14 to 17 nt from the cleavage site (Chanfreau et al., 1998a). The structure surrounding the 3' ETS cleavage site corresponds to this consensus and the newly identified sites of cleavage are located at the expected distance from the closing tetraloop (see Fig. 7B). In contrast, the structure within the $5^{\prime}$ ETS is not closed by a tetraloop and the loop is much further away from the cleavage site (40 nt) (Yeh \& Lee, 1992). Moreover, deletion of the loop region from the $5^{\prime}$ ETS stem-loop structure did not 
detectably inhibit $A_{0}$ cleavage (Venema et al., 1995). In fact, the model 5' ETS substrate was apparently a poor in vitro substrate for recombinant Rnt1p; the in vitro assay for cleavage of the 5' ETS was performed using a higher enzyme concentration than was used for a model 3' ETS substrate (Abou Elela et al., 1996). Moreover, both assays were performed with an excess of enzyme over substrate, which may have led to nonspecific cleavage at $A_{0}$ and inaccurate cleavage of the $3^{\prime}$ ETS. Less clear is why the rnt1-1 mutation inhibited in vivo processing whereas the complete deletion of the gene did not. A possible explanation is that the rnt1-1 mutation fortuitously conferred a dominant negative phenotype. It remains possible that Rnt1p does have a role in $A_{0}$ cleavage, but, if so, there must be another endonuclease that cleaves the same site.

The only component currently known to be required for cleavage at site $A_{0}$ is the U3 (Hughes \& Ares, 1991). This function requires base pairing between the U3 snoRNA and a sequence in the pre-rRNA 5' ETS that lies 130 nt upstream of $A_{0}$ (Beltrame \& Tollervey, 1992, 1995). It was proposed that the U3/5' ETS interaction targets the endonuclease to its cleavage site (Abou Elela et al., 1996). This remains an attractive model, but an endonuclease other than Rnt1p must now be sought.

\section{MATERIALS AND METHODS}

\section{Strains}

Growth and handling of S. cerevisiae were by standard techniques. The transformation procedure was according to Gietz et al. (1992).

Two wild-type RNT1 strains (spores a and b) and two isogenic rnt1- $\Delta$ strains (spores $c$ and d) derived from a single dissected tetrad (Chanfreau et al., 1998b) were generously provided by G. Chanfreau. Strain rat1-1 (MAT $\alpha$, his3- $\Delta 200$; leu2- $\Delta$; ura3-52, rat1-1) was kindly provided by C. Cole (Amberg et al., 1992). Strain JH84 (MATa; leu2-3,12; ura352; his3- 200 , ade2-1; CAN1-100; GAL10::U3; UAS ${ }_{G A L}::$ SNR17A-URA3; SNR17B::LEU2) was kindly provided by J. Hughes (Hughes \& Ares, 1991). Strain YDL401 (MATa; ura3-

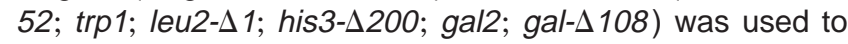
construct GAL::rnt1 strain using a one-step PCR strategy (Lafontaine \& Tollervey, 1996). Oligonucleotides 1, 5'-TGCG GCCCTAAACCATGCTGAAATTTTTCATGAGCTCATCGCA AAACTCTTGGCCTCCTCTTAG and 2, 5'-ATTATCATTCTG GGTTTTCTTTTTACCTGCTACTTTTGAGCCCATATGGTG ATGGTGATGGTGAT GGTGAGA were used for the amplification with plasmid pTL32. Transformants were selected for $\mathrm{His}^{+}$prototrophy and were screened by PCR and Southern blot analysis. The GAL::His ${ }_{8}$-RNT1 construct supported wildtype growth on RSG medium at $30^{\circ} \mathrm{C}$.

\section{RNA extraction, Northern hybridization, and primer extension}

For Rnt1p depletion cells were harvested at intervals following the shift from RSG medium ( $2 \%$ galactose, $2 \%$ sucrose,
$2 \%$ raffinose) to medium containing $2 \%$ glucose. RNA was extracted as described previously (Tollervey \& Mattaj, 1987). Northern hybridization (Tollervey, 1987) and primer extension (Beltrame \& Tollervey, 1992) were as described previously. Standard $1.2 \%$ agarose/formaldehyde and $6 \%$ or $8 \%$ acrylamide gels were used to analyze the high and low molecular weight rRNA species and primer extension reactions.

For pre-rRNA hybridization and primer extension, oligonucleotides depicted in Figure $1 \mathrm{~A}$ were used:

001, 5'-CCAGTTACGAAAATTCTTG; 002, 5'-GCTCTTTGCTCTTGCC; 005, 5'-ATGAAAACTCCACAGTG; 007, 5'-CTCCGCTTATTGATATGC; 008, 5'-CATGGCTTAATCTTTGAGAC; 013, 5'-GGCCAGCAATTTCAAGTTA; 015, 5'-TTTCGCTGCGTTCTTCATC; 022, 5'-GAAATAAAAAACAAATCAGAC; 026; 5'-CCAGATAACTATCTTAAAAG; 053, 5'-TGGTACACTCTTACACAC; 054, 5'-AACCCATCTTTGCAACGA.

For detection of the excised $A_{0}-A_{1}$ fragment, a riboprobe complementary to the region between sites $A_{0}$ and $A_{1}$ was generated as previously described (Venema et al., 1995). For hybridization to RNT1, mRNA oligonucleotide anti-RNT1 was used, 5'-GCCTTTGTTGGGTCATAGCTATCTTCTCCT TCGTC.

\section{ACKNOWLEDGMENTS}

We thank Guillaume Chanfreau for providing the RNT1 and rnt1- $\Delta$ strains, and for useful discussions, and Christine Allmang, Phil Mitchell, and Denis Lafontaine for critical reading of the manuscript. J.K. was the recipient of an EMBO fellowship. This work was supported by the Wellcome Trust.

Received February 12, 1999; returned for revision March 5, 1999; revised manuscript received April 7, 1999

\section{REFERENCES}

Abou Elela S, Ares MJ. 1998. Depletion of yeast RNase III blocks correct U2 $3^{\prime}$ end formation and results in polyadenylated but functional U2 snRNA. EMBO J 17:3738-3746.

Abou Elela S, Igel H, Ares MJ. 1996. RNase III cleaves eukaryotic preribosomal RNA at a U3 snoRNP-dependent site. Cell 85:115124.

Allmang C, Tollervey D. 1998. The role of the 3' external transcribed spacer in yeast pre-rRNA processing. $\mathrm{J} \mathrm{Mol} \mathrm{Biol} \mathrm{278:67-78.}$

Altman S, Kirsebom L, Talbot S. 1993. Recent studies of ribonuclease P. FASEB J 7:7-14.

Amberg DC, Goldstein AL, Cole CN. 1992. Isolation and characterization of RAT1: An essential gene of Saccharomyces cerevisiae required for the efficient nucleocytoplasmic trafficking of mRNA. Genes \& Dev 6:1173-1189.

Beltrame M, Tollervey D. 1992. Identification and functional analysis of two U3 binding sites on yeast pre-ribosomal RNA. EMBO J 11:1531-1542.

Beltrame M, Tollervey D. 1995. Base pairing between U3 and the pre-ribosomal RNA is required for $18 \mathrm{~S}$ rRNA synthesis. EMBO J 14:4350-4356.

Bergès T, Petfalski E, Tollervey D, Hurt EC. 1994. Synthetic lethality with fibrillarin identifies NOP77p, a nucleolar protein required for pre-rRNA processing and modification. EMBO J 13:3136-3148. 
Chanfreau G, Abou Elela S, Ares M Jr, Guthrie C. 1997. Alternative 3 '-end processing of U5 snRNA by RNase III. Genes \& Dev 11:2741-2751.

Chanfreau G, Legrain P, Jacquier A. 1998a. Yeast RNase III as a key processing enzyme in small nucleolar RNAs metabolism. $J$ Mol Biol 248:975-988.

Chanfreau G, Rotondo G, Legrain P, Jacquier A. 1998b. Processing of a dicistronic small nucleolar RNA precursor by the RNA endonuclease Rnt1. EMBO J 17:3726-3737.

Court D. 1993. RNAse III: A double-strand processing enzyme. In: Brawerman G, Belasco J, eds. Control of mRNA stability. New York: Academic Press. pp 70-116.

Daugeron MC, Linder P. 1998. Dbp7p, a putative ATP-dependent RNA helicase from Saccharomyces cerevisiae, is required for $60 \mathrm{~S}$ ribosomal subunit assembly. RNA 4:566-581.

de la Cruz J, Kressler D, Rojo M, Tollervey D, Linder P. 1998a. $\mathrm{Spb} 4 \mathrm{p}$, an essential putative RNA helicase, is required for a late step in the assembly of 60S ribosomal subunits in Saccharomyces cerevisiae. RNA 4:1268-1281.

de la Cruz J, Kressler D, Tollervey D, Linder P. 1998b. Dob1p (Mtr4p) is a putative ATP-dependent RNA helicase required for the $3^{\prime}$ end formation of 5.8S rRNA in Saccharomyces cerevisiae. EMBO J 17:1128-1140.

Gietz D, St Jean A, Woods RA, Schiestl RH. 1992. Improved method for high efficient transformation of intact yeast cells. Nucleic Acids Res 20:1425.

Hughes JMX, Ares MJ. 1991. Depletion of U3 small nucleolar RNA inhibits cleavage in the $5^{\prime}$ external transcribed spacer of yeast pre-ribosomal RNA and impairs formation of $18 \mathrm{~S}$ ribosomal RNA. EMBO J 10:4231-4239.

Kempers-Veenstra AE, Oliemans J, Offenberg H, Dekker AF, Piper PW, Planta RJ, Klootwijk J. 1986. 3'-end formation of transcripts from the yeast rRNA operon. EMBO J 5:2703-2710.

King TC, Sirdeshmukh R, Schlessinger D. 1984. RNase III cleavage is obligate for maturation but not for function of Escherichia coli pre-23S rRNA. Proc Natl Acad Sci USA 81:185-188.

Kressler D, de la Cruz J, Rojo M, Linder P. 1998. Dbp6p is an essential putative ATP-dependent RNA helicase required for 60Sribosomal-subunit assembly in Saccharomyces cerevisiae. Mol Cell Biol 18:1855-1865.

Lafontaine D, Tollervey D. 1996. One-step PCR mediated strategy for the construction of conditionally expressed and epitope tagged yeast proteins. Nucleic Acids Res 24:3469-3472.

Lang WH, Reeder RH. 1993. The REB1 site is an essential component of a terminator for RNA polymerase I in Saccharomyces cerevisiae. Mol Cell Biol 13:649-658.

Li HV, Zagorski J, Fournier MJ. 1990. Depletion of U14 small nuclear
RNA (snR128) disrupts production of 18S rRNA in Saccharomyces cerevisiae. Mol Cell Biol 10:1145-1152.

Lygerou Z, Allmang C, Tollervey D, Séraphin B. 1996. Accurate processing of a eukaryotic precursor ribosomal RNA by ribonuclease MRP in vitro. Science 222:268-270.

Petfalski E, Dandekar T, Henry Y, Tollervey D. 1998. Processing of the precursors to small nucleolar RNAs and rRNAs requires common components. Mol Cell Biol 18:11181-11189.

Qu LH, Henras A, Lu YJ, Zhou H, Zhou W, Zhu YQ, Zhao J, Henry Y, Caizergues-Ferrer M, Bachellerie JP. 1999. Seven novel methylation guide small nucleolar RNAs are processed from a common polycistronic transcript by Rat1p and RNase III in yeast. Moll Cell Biol 19:1144-1158.

Seipelt RL, Zheng B, Asuru A, Rymond BC. 1999. U1 snRNA is cleaved by RNase III and processed through an Sm site-dependent pathway. Nucleic Acids Res 27:587-595.

Tollervey D. 1987. A yeast small nuclear RNA is required for normal processing of pre-ribosomal RNA. EMBO J 6:4169-4175.

Tollervey D, Mattaj IN. 1987. Fungal small nuclear ribonucleoproteins share properties with plant and vertebrate u-su RNAs. EMBO $J$ 6:469-476.

van der Sande CAFM, Kulkens T, Kramer AB, de Wijs IJ, van Heerikhuizen H, Klootwijk J, Planta RJ. 1989. Termination of transcription by yeast RNA polymerase I. Nucleic Acids Res 17:9127-9146.

Veldman GM, Klootwijk J, De Jong P, Leer RJ, Planta RJ. 1980. The transcription termination site of the ribosomal RNA operon in yeast. Nucleic Acids Res 8:5179-5192.

Veldman GM, Klootwijk J, van Heerikhuizen H, Planta RJ. 1981. The nucleotide sequence of the intergenic region between the $5.8 \mathrm{~S}$ and 26S rRNA genes of the yeast ribosomal RNA operon. Possible implications for the interaction between 5.8S and 26S rRNA and the processing of the primary transcript. Nucleic Acids Res 9:4847-4862.

Venema J, Henry Y, Tollervey D. 1995. Two distinct recognition signals define the site of endonucleolytic cleavage at the $5^{\prime}$ end of yeast $18 \mathrm{~S}$ rRNA. EMBO J 14:4883-4892.

Venema J, Tollervey D. 1995. Processing of pre-ribosomal RNA in Saccharomyces cerevisiae. Yeast 11:1629-1650.

Weaver PL, Sun C, Chang T-H. 1997. Dbp3p, a putative RNA helicase in Saccharomyces cerevisiae, is required for efficient pre-rRNA processing predominantly at site $\mathrm{A}_{3}$. Mol Cell Biol 17:1354-1365.

Yeh L-CC, Lee JC. 1992. Structure analysis of the $5^{\prime}$ external transcribed spacer of the precursor ribosomal RNA from Saccharomyces cerevisiae. J Mol Biol 228:827-839.

Zanchin NIT, Roberts P, DeSilva A, Sherman F, Goldfarb DS. 1997. Saccharomyces cerevisiae Nip7p is required for efficient 60S ribosome biogenesis. Mol Cell Biol 17:5001-5015. 

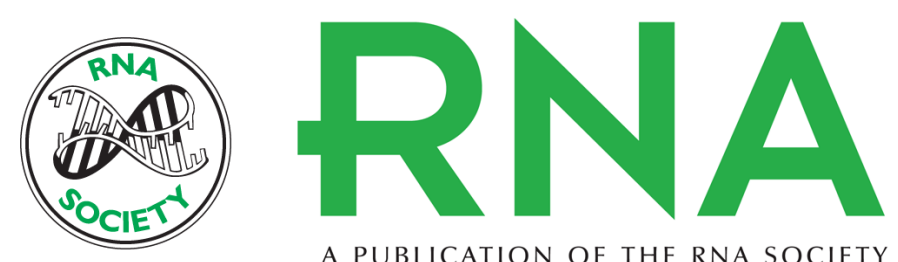

A PUBLICATION OF THE RNA SOCIETY

\section{Yeast Rnt1p is required for cleavage of the pre-ribosomal RNA in the 3' ETS but not the 5' ETS.}

J Kufel, B Dichtl and D Tollervey

RNA 1999 5: 909-917

\section{License}

Email Alerting Receive free email alerts when new articles cite this article - sign up in the box at the Service top right corner of the article or click here. 